\title{
PET/CT-Derived Whole-Body and Bone Marrow Dosimetry of ${ }^{89} \mathrm{Zr}$-Cetuximab
}

\author{
Nikolaos E. Makris ${ }^{1}$, Ronald Boellaard ${ }^{1}$, Arthur van Lingen ${ }^{1}$, Adriaan A. Lammertsma ${ }^{1}$, Guus A.M.S. van Dongen ${ }^{1}$, \\ Henk M. Verheul' ${ }^{2}$, Catharina W. Menke ${ }^{2}$, and Marc C. Huisman ${ }^{1}$ \\ ${ }^{I}$ Department of Radiology and Nuclear Medicine, VU University Medical Center, Amsterdam, The Netherlands; and ${ }^{2}$ Department of \\ Medical Oncology, VU University Medical Center, Amsterdam, The Netherlands
}

PET/CT imaging allows for image-based estimates of organ and red marrow (RM) residence times. The aim of this study was to derive $\mathrm{PET} / \mathrm{CT}$-based radiation dosimetry for ${ }^{89} \mathrm{Zr}$-cetuximab, with special emphasis on determining RM-absorbed dose. Methods: Seven patients with colorectal cancer received $36.9 \pm 0.8 \mathrm{MBq}$ of ${ }^{89} \mathrm{Zr}$ cetuximab within $2 \mathrm{~h}$ after administration of a therapeutic dose of $500 \mathrm{mg} \cdot \mathrm{m}^{-2}$ of cetuximab. Whole-body PET/CT scans and blood samples were obtained at $1,24,48,94$, and $144 \mathrm{~h}$ after injection. $\mathrm{RM}$ activity concentrations were calculated from manual delineation of the lumbar vertebrae and blood samples, assuming a fixed RMto-plasma activity concentration ratio (RMPR) of 0.19 . The cumulated activity was calculated as the area under the curve of the organ time-activity data (liver, lungs, kidneys, spleen, and RM), assuming physical decay after the last scan. The residence time for each organ was derived by dividing the cumulated activity with the total injected activity. The residence time in the remainder of the body was calculated as the maximum possible residence time minus the sum of residence time of source organs, assuming no excretion during the time course of the scans. The (self and total) $\mathrm{RM}$ - and organ-absorbed doses and effective whole-body radiation dose were obtained using dose conversion factors from OLINDA/ EXM 1.1. Several simplified 3-time-point dosimetry approaches were also evaluated. Results: The first approach yielded self and total RM doses of $0.17 \pm 0.04$ and $0.51 \pm 0.06 \mathrm{mGy} \cdot \mathrm{MBq}^{-1}$, respectively. The second approach deviated by $-21 \%$ in self-dose and $-6 \%$ in total dose. RMPR increased over time in 5 of 7 patients. The highest ${ }^{89} \mathrm{Zr}$-absorbed dose was observed in the liver with $2.60 \pm$ $0.78 \mathrm{mGy} \cdot \mathrm{MBq}^{-1}$, followed by the kidneys, spleen, and lungs, whereas the effective whole-body dose was $0.61 \pm 0.09$ $\mathrm{mSv} \cdot \mathrm{MBq}^{-1}$. The simplified 3-time-point (1, 48, and $\left.144 \mathrm{~h}\right)$ dosimetry approach deviated by at most $4 \%$ in both organ-absorbed doses and effective dose. Conclusion: Although the total RM dose estimates obtained with the 2 approaches differed only by at most $6 \%$, the image-based approach is preferred because it accounts for nonconstant RMPR. The number of successive scans can be reduced to 3 without affecting effective dose estimates.

Key Words: ${ }^{89} \mathrm{Zr}$; PET; cetuximab; red marrow; absorbed dose

J Nucl Med 2015; 56:249-254

DOI: 10.2967/jnumed.114.147819

Received Aug. 30, 2014; revision accepted Dec. 15, 2014.

For correspondence or reprints contact: Nikolaos Makris, VU University Medical Center, Department of Radiology and Nuclear Medicine, De Boelelaan 1117, 1081 HV Amsterdam, The Netherlands.

E-mail: n.makris@vumc.nl

Published online Jan. 22, 2015.

COPYRIGHT (c) 2015 by the Society of Nuclear Medicine and Molecular Imaging, Inc.
$\mathbf{P}$ tool for predicting the biodistribution of labeled monoclonal antibodies (mAbs) $(1,2)$ and organ dosimetry for radioimmunotherapy (2). In addition, the dose-limiting tissue can be determined, enabling dose escalation and optimization of therapeutic treatment planning. In particular, a recent study showed that the biodistributions of ${ }^{89} \mathrm{Zr}$ Df-cetuximab and ${ }^{88}$ Y-DOTA-cetuximab $\left({ }^{88} \mathrm{Y}\right.$ as a substitute for ${ }^{90} \mathrm{Y}$ ) were comparable for all organs (1). Another study from the same group demonstrated nearly identical biodistributions of ${ }^{89} \mathrm{Zr}$ ibritumomab and ${ }^{90}$ Y-ibritumomab (2). Recently, the effect of radioimmunotherapy using ${ }^{90}$ Y-cetuximab (combined with external-beam irradiation) on local tumor control in vivo was examined in 3 human squamous cell carcinoma models (3). The latter study showed that PET imaging using ${ }^{86}$ Y-cetuximab may be used to assess epidermal growth factor receptor expression, which in turn could be a potential predictor for response to combined radioimmunotherapy and externalbeam radiotherapy.

With radioimmunotherapy, bone marrow can be the dose-limiting organ. Conventionally, the red marrow (RM) activity concentration is assumed to be $19 \%$ of the plasma activity concentration (4). Assuming a hematocrit value of 0.44 , the red marrow (RM)-to-blood ratio (RMBLR) will be assigned a value of 0.34 . However, recent studies by Schwartz et al. (5) and Hindorf et al. (6) have reported a timedependent RM-to-plasma ratio (RMPR) based on PET imaging using ${ }^{124} \mathrm{I}-\mathrm{cG} 250$ and ${ }^{124} \mathrm{I}-\mathrm{huA} 33$ and scintigraphic imaging using ${ }^{131} \mathrm{I}-$ labeled anti-CD22 mAb, respectively. This increase in RMPR may reflect binding to Fc receptor-expressing cells in bone marrow. Those observations imply that RM dose estimates based on blood or plasma activity concentrations may be inappropriate, at least for some mAbs. Schwartz et al. (5) reported that the plasma-based approach can produce discrepancies of as much as -74 to $+62 \%$ in individual patients for self RM dose (after ${ }^{124} \mathrm{I}$-labeled mAb administration), as compared with PET/CT image-based dosimetry. It has also been reported that ${ }^{124} \mathrm{I}$-labeled $\mathrm{mAbs}$ tend to release free radionuclides on antibody internalization, resulting in rapid clearance of the radionuclides from the target tissue, leading to reduced tumor contrast (7) and a change in RMPR over time. Unlike ${ }^{124} \mathrm{I},{ }^{89} \mathrm{Zr}$ appears to be a residualizing radiometal potentially circumventing these problems (7). However, increased radioactivity in bone, as reported in recent studies $(8,9)$ using ${ }^{89} \mathrm{Zr}$ as a PET tracer, has not been analyzed adequately yet to assess whether or not in vivo metal release or other mechanisms are involved. Again, a consequence could be that the assumption of a constant RMPR is wrong.

The novelty of this study lies in the exploration of the added potential of performing a PET/CT-derived biodistribution/dosimetry 
TABLE 1

Patient Details

Residence time Whole-body-to-blood in blood (h) cumulated activity ratio

\begin{tabular}{llll}
\hline M & 72 & 44 & 2.1 \\
M & 82 & 47 & 2.1 \\
M & 79 & 35 & 2.5 \\
M & 79 & 26 & 3.4 \\
\hline F & 75 & 43 & 2.2 \\
\hline F & 93 & 40 & 2.5 \\
\hline F & 69 & 56 & 1.8 \\
\hline
\end{tabular}

study in humans for a mAb labeled with a positron emitter. The advantage of the associated (low-dose) CT scan is more robust organ delineation. In addition, use of a CT-defined volume of interest (VOI) of the lumbar vertebrae (LV) may allow for noninvasive quantification of RM activity concentrations. The aim of this study was to assess biodistribution and radiation dosimetry of ${ }^{89} \mathrm{Zr}$-cetuximab in humans, with a special emphasis on a comparison of image- and plasma-based RM dose-estimation approaches.

TABLE 2

Parameter Overview

\begin{tabular}{|c|c|}
\hline Parameter & Definition \\
\hline$\left[\tilde{A}_{R M}\right]$ & $\begin{array}{l}\text { Cumulated activity concentration in } \\
\text { the RM }\end{array}$ \\
\hline$\left[\tilde{A}_{P L}\right]$ & $\begin{array}{l}\text { Cumulated activity concentration in } \\
\text { the plasma }\end{array}$ \\
\hline$\tilde{A}_{R M}$ & Cumulated activity in the RM \\
\hline$\tilde{A}_{W B}$ & Cumulated activity in the whole body \\
\hline$\tilde{A}_{R B}$ & $\begin{array}{l}\text { Cumulated activity in the remainder } \\
\text { body }\end{array}$ \\
\hline RMPR & $\begin{array}{l}\text { RM-to-plasma activity concentration } \\
\text { ratio }\end{array}$ \\
\hline RMECFF & $\begin{array}{l}\text { RM-to-extracellular fluid activity } \\
\text { concentration fraction }\end{array}$ \\
\hline$m_{R M-p a t i e n t}$ & Patient-specific RM mass \\
\hline$m_{W B-p a t i e n t}$ & Patient-specific whole-boy mass \\
\hline$m_{R M-M I R D O S E 3}$ & Standard RM mass \\
\hline$m_{\text {WB-MIRDOSE3 }}$ & Standard whole-body mass \\
\hline$D_{R M}^{\text {Self }}$ & Self RM dose \\
\hline$D_{R M}^{\text {Cross }}$ & Cross RM dose \\
\hline$D_{R M}^{\text {Total }}$ & Total RM dose \\
\hline$S(R M \leftarrow R M)$ & $\begin{array}{l}\text { Dose conversion factor for } \mathrm{RM} \text { to } \mathrm{RM} \\
\text { contribution }\end{array}$ \\
\hline$S(R M \leftarrow R B)$ & $\begin{array}{l}\text { Dose conversion factor for remainder } \\
\text { body to RM contribution }\end{array}$ \\
\hline$S(R M \leftarrow W B)$ & $\begin{array}{l}\text { Dose conversion factor for whole-body } \\
\text { to RM contribution }\end{array}$ \\
\hline $\mathrm{HU}$ & Hounsfield unit \\
\hline
\end{tabular}

\section{MATERIALS AND METHODS}

\section{Imaging Protocol}

Seven patients (4 men, 3 women) with histopathologically confirmed advanced kRas wild-type colorectal cancer (Table 1) received $36.9 \pm$ $0.8 \mathrm{MBq}$ of ${ }^{89} \mathrm{Zr}$-cetuximab within $2 \mathrm{~h}$ after administration of the first therapeutic dose of $500 \mathrm{mg} \cdot \mathrm{m}^{-2}$ of cetuximab. PET/CT scans (Gemini TF-64; Philips Healthcare) and blood samples were obtained at 1, 24, 48, 94, and $144 \mathrm{~h}$ after injection (10). PET data were normalized; corrected for decay, randoms, dead time, scatter, and attenuation; and reconstructed using a time-of-flight list-mode ordered-subsets expectation maximization reconstruction method with a matrix size of $144 \times 144$ and a voxel size of $4 \times 4 \times 4 \mathrm{~mm}^{3}$. In addition, for each time point, a 50-mA low-dose $\mathrm{CT}$ scan was acquired for attenuation-correction purposes. Corresponding CT images were reconstructed with an image matrix size of $512 \times 512$ and a voxel size of $1.17 \times 1.17 \times 5 \mathrm{~mm}^{3}$. For the present analysis, all 5 CT scans for each patient were rebinned into a $4 \times 4 \times 4 \mathrm{~mm}^{3}$ voxel size to map CT VOIs onto the PET images. The study was approved by the Medical Ethics Committee of the VU University Medical Center, and all patients signed a written informed consent form before the inclusion.

\section{Organ Dosimetry}

The activity for each organ that was visible in all PET scans (liver, lungs, kidneys, spleen, and RM) was determined using the mean activity concentration in VOIs with in-house-developed software. VOIs were independently drawn on all 5 CT scans for each patient and subsequently mapped onto the respective PET scans. Total organ activities were derived using standard organ masses as reported by Stabin et al. (11). The cumulated activity was calculated as the area under the curve of the organ timeactivity data approximated by the trapezoidal rule and assuming only physical decay after the last measurement. Next, the residence time was derived by dividing the cumulated activity by the total injected activity. The residence time in the remainder of the body was calculated as the maximum residence time (based on physical decay only) minus the sum of residence time of source organs (an organ was designated as source organ when uptake was visible), assuming no excretion during the time course of the scans. Although the effective total residence time could also be derived

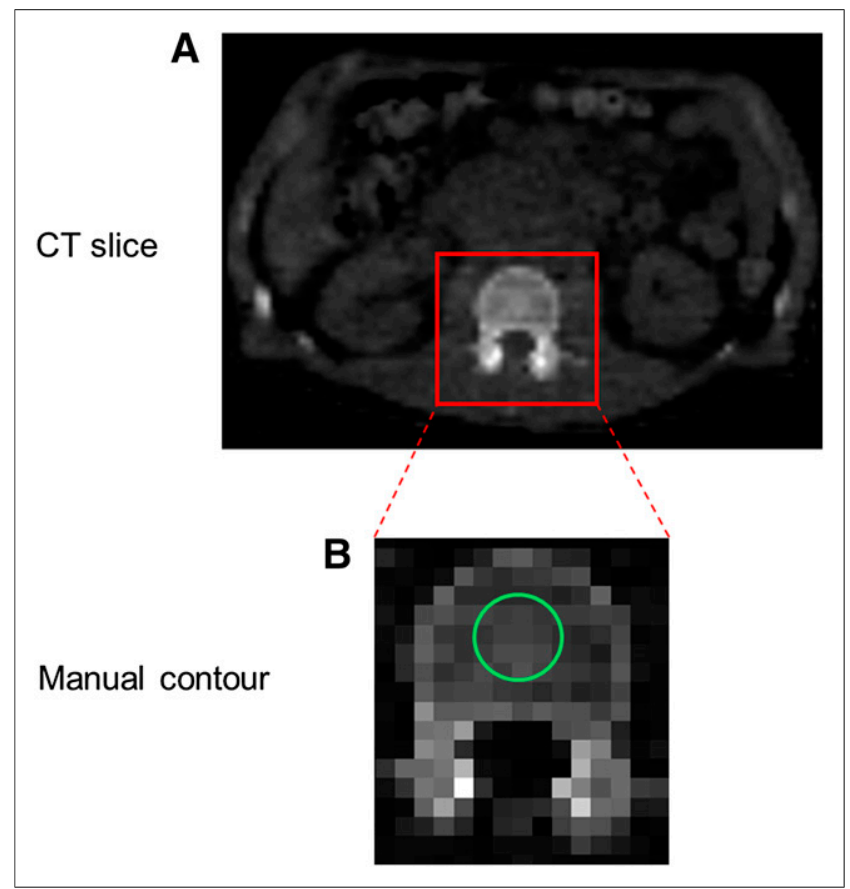

FIGURE 1. Original CT slice (A) and axial CT slice (B) with manually defined LV contour (green line) enclosing intraosseous volume. 


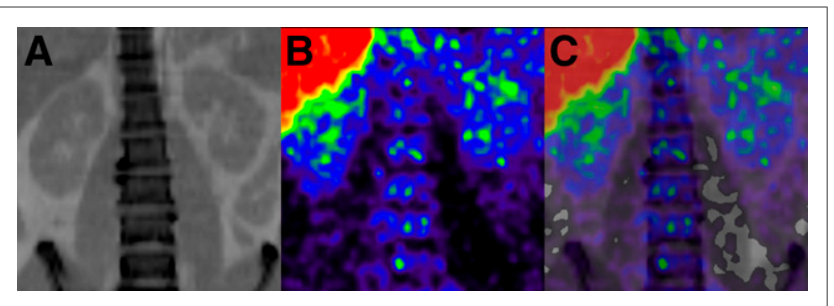

FIGURE 2. Typical example of coronal slices of CT (A), PET (B), and PET/CT (C).

from a whole-body VOI (on average 35\% lower values), this approach was not followed to obtain conservative estimates of the effective dose. Individual residence times were scaled with the mass ratio of the patient to reference man/woman before being used as input in OLINDA/EXM 1.1. This software was used for the calculation of organ-absorbed doses and effective dose (11). To derive a simplified dosimetry protocol with 3 time points, all possible combinations were tested for the ability to estimate organ-absorbed doses and effective doses as accurately as possible.

\section{RM Dose-Estimation Methods}

Blood-Based Method. Conventionally, the blood-based approach assumes that plasma activity concentration is equal to the extracellular fluid activity concentration in the marrow space and, therefore, that RMPR is constant, equal to the fraction of RM composed of extracellular fluid (RMECFF) (4). In this method, a fixed, time-independent RMPR value of 0.19 is used. In Table 2, a parameter overview can be found. Plasma samples were counted in a Wallac 1470 well counter (Perkin Elmer Lifescience), and conversion of the derived counts per minute to disintegration per minute was done (a description of the methodology of cross calibration between the PET scanner and the well counter can be found in Greuter et al. (12)). The total cumulated activity concentration in the RM is given by:

$$
\left[\tilde{A}_{R M}\right]=\operatorname{RMECFF} \times\left[\tilde{A}_{P L}\right]
$$

or alternatively the cumulated activity can be written as:

$$
\tilde{A}_{R M}=\operatorname{RMECFF} \times\left[\tilde{A}_{P L}\right] \times m_{R M-\text { patient }} .
$$

The RM mass can be approximated through the standard adult and patient-specific whole-body mass:

$$
\begin{gathered}
m_{R M-\text { patient }}=\frac{m_{R M-M I R D O S E 3}}{m_{W B-M I R D O S E 3}} \times m_{W B-\text { patient }} \\
\tilde{A}_{R M}=\operatorname{RMECFF} \times\left[\tilde{A}_{P L}\right] \times \frac{m_{R M-M I R D O S E 3}}{m_{W B-M I R D O S E 3}} \times m_{W B-p a t i e n t},
\end{gathered}
$$

where $m_{R M-M I R D O S E 3}, m_{W B}-$ MIRDOSE3 , and $m_{W B}$ - patient correspond to the standard adult mass for RM (men, $1.12 \mathrm{~kg}$; women, $1.30 \mathrm{~kg}$ ), whole body (men, $73.7 \mathrm{~kg}$; women, $58.0 \mathrm{~kg}$ ) (11), and the patient-specific whole-body mass, respectively (Table 2 ). The total RM-absorbed dose can be divided into 2 contributions, the self RM dose-which represents the dose from the marrow spaces-and the cross RM dosewhich represents the dose from the remaining tissues of the body $(13,14)$. This dose can be expressed by the following equations:

$$
\begin{gathered}
D_{R M}^{\text {Total }}=D_{R M}^{\text {Self }}+D_{R M}^{\text {Cross }} \\
D_{R M}^{\text {Total }}=\tilde{A}_{R M} \times S(R M \leftarrow R M)+\left(\tilde{A}_{W B}-\tilde{A}_{R M}\right) \times S(R M \leftarrow R B)
\end{gathered}
$$

The full expressions of self-dose and cross-dose contribution to the RM can be obtained by substituting Equations 2, 3, and 4 into Equation
6. By introducing a mass scaling for the $\mathrm{S}$ factors in Equation 6, the $m_{W B}$-patient terms cancel out and a patient mass-independent term remains, whereas the final cross RM dose term will be patient massdependent. Calculations and full expression of the formulas can be found in the supplemental materials (available at http://jnm.snmjournals.org).

Manual VOI Delineation Method. In immuno-PET studies, a second approach to determine $\left[\tilde{A}_{R M}\right]$ is by delineating VOIs in each of the 5 (L1-L5) segments of the LV on CT slices (Fig. 1). Each VOI had a spheric shape with a volume of $6 \mathrm{~mL}$, providing a total volume of $30 \mathrm{~mL}$ for all 5 segments. Subsequently, all 5 VOIs were transferred to the PET images, and the mean activity concentration was calculated. The effect of using smaller or larger volumes in estimating mean activity concentration was also investigated. The LV consists of compact bone, trabecular bone, and marrow space elements - that is, red and yellow marrow, extracellular fluid, and vasculature. Assuming that there is no specific binding of the radiolabeled antibody cetuximab to trabecular bone, it follows that the trabecular bone activity concentration should be zero. Thus, a correction factor was applied for the presence of trabecular bone in the LV segments. To this end, the RM activity concentration was scaled on the basis of the volume of the LV composed of trabecular bone ( $\mathrm{f}_{\mathrm{tb}}$; men, 0.135 ; women, 0.148 ) (15), thus a multiplicative correction factor $\left(1 /\left(1-\mathrm{f}_{\mathrm{tb}}\right)\right)$ was applied. This approach does not assume a constant RMPR over time as it is an image-derived method. Equation 4 was adjusted by replacing $\left[\tilde{A}_{P L}\right] \times \mathrm{RMECFF}$ with $\left[\tilde{A}_{R M}\right]$, as the RM activity concentration was directly obtained from the PET images. Visual inspection of the PET images did not show higher uptake in the compact bone component when compared with the marrow space elements of the LV (Fig. 2).

\section{RESULTS}

Figure 3 shows RMPR as a function of time for patients injected with ${ }^{89} \mathrm{Zr}$-cetuximab. RMPR at the time of the first scan $(1 \mathrm{~h})$ was $0.13 \pm 0.03$ (range, $0.09-0.16$ ), whereas for the last scans (144 h) an increased RMPR of $0.49 \pm 0.29$ (range, 0.22-0.99) was observed. While varying the volumes used in the bone marrow of the $\mathrm{LV}$, we obtained bone marrow activity concentration that deviated, at most $7 \%$, when compared with $\mathrm{AC}_{\mathrm{RM}}$ obtained from $30-\mathrm{mL}$

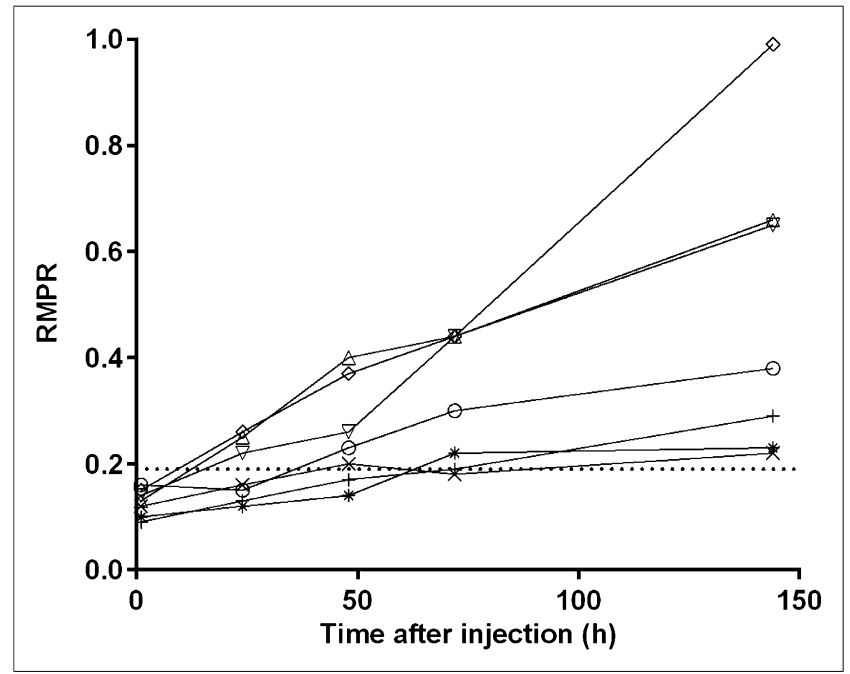

FIGURE 3. Image-derived RMPR as function of imaging time after injection of ${ }^{89} \mathrm{Zr}$-cetuximab. Five of 7 patients depict increasing RMPR as function of time, and only in 2 patients RMPR corresponds with nominal value of 0.19 (dotted line). 


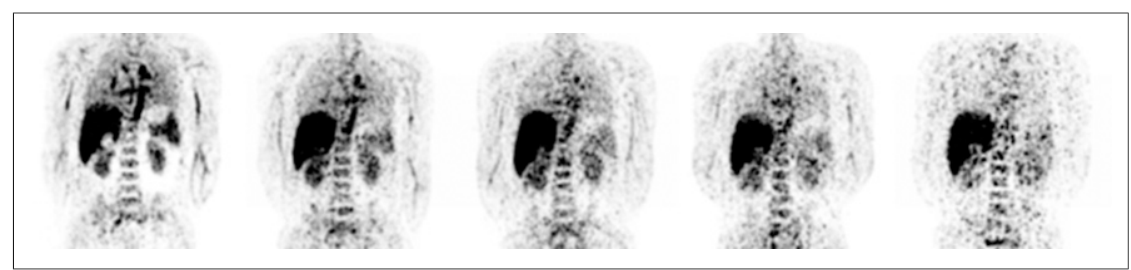

FIGURE 4. Biodistribution of ${ }^{89} \mathrm{Zr}$-cetuximab as visualized using PET during course of $7 \mathrm{~d}$ (left to right: $1,24,48,72$, and $144 \mathrm{~h}$ after injection).

bone marrow volumes. Typical coronal slices of ${ }^{89} \mathrm{Zr}$-cetuximab images during the time course of $7 \mathrm{~d}$ can be seen in Figure 4.

The self RM dose estimate as calculated for the plasma-based approach was $0.13 \pm 0.05 \mathrm{mGy} \cdot \mathrm{MBq}^{-1}$ (range, 0.08-0.24, Fig. 5). The LV-based self RM dose estimate was $0.17 \pm 0.04 \mathrm{mGy} \cdot \mathrm{MBq}^{-1}$ (range, $0.11-0.22 \mathrm{mGy} \cdot \mathrm{MBq}^{-1}$ ). The total $\mathrm{RM}$ dose estimate for the plasma- and LV-based approaches was $0.48 \pm 0.08 \mathrm{mGy} \cdot \mathrm{MBq}^{-1}$ (range, $0.41-0.65 \mathrm{mGy} \cdot \mathrm{MBq}^{-1}$ ) and $0.51 \pm 0.06 \mathrm{mGy} \cdot \mathrm{MBq}^{-1}$ (range, $0.44-0.63 \mathrm{mGy} \cdot \mathrm{MBq}^{-1}$ ), respectively (Table 3 ). The contribution of cumulated activity before the first and after the last scan as compared with the total RM cumulated activity was $16 \% \pm 2 \%$ and $27 \% \pm 4 \%$ for plasma- and LV-based methods, respectively. In addition, across all patients, the self RM dose percentage contribution to the total RM dose varied from $18 \%$ to $35 \%$, whereas the whole-bodyto-blood cumulated activity ratio varied from 3.4 to 1.8 .

The organ average uptake for liver, lungs, kidneys, spleen, and RM is shown in Figure 6. The highest average absorbed dose was observed in the liver with $2.60 \pm 0.78 \mathrm{mGy} \cdot \mathrm{MBq}^{-1}$, followed by the kidneys $\left(1.04 \pm 0.24 \mathrm{mGy} \cdot \mathrm{MBq}^{-1}\right)$, spleen $\left(0.89 \pm 0.22 \mathrm{mGy} \cdot \mathrm{MBq}^{-1}\right)$, lungs $\left(0.66 \pm 0.17 \mathrm{mGy} \cdot \mathrm{MBq}^{-1}\right)$, and $\mathrm{RM}\left(0.51 \pm 0.06 \mathrm{mGy} \cdot \mathrm{MBq}^{-1}\right)$.

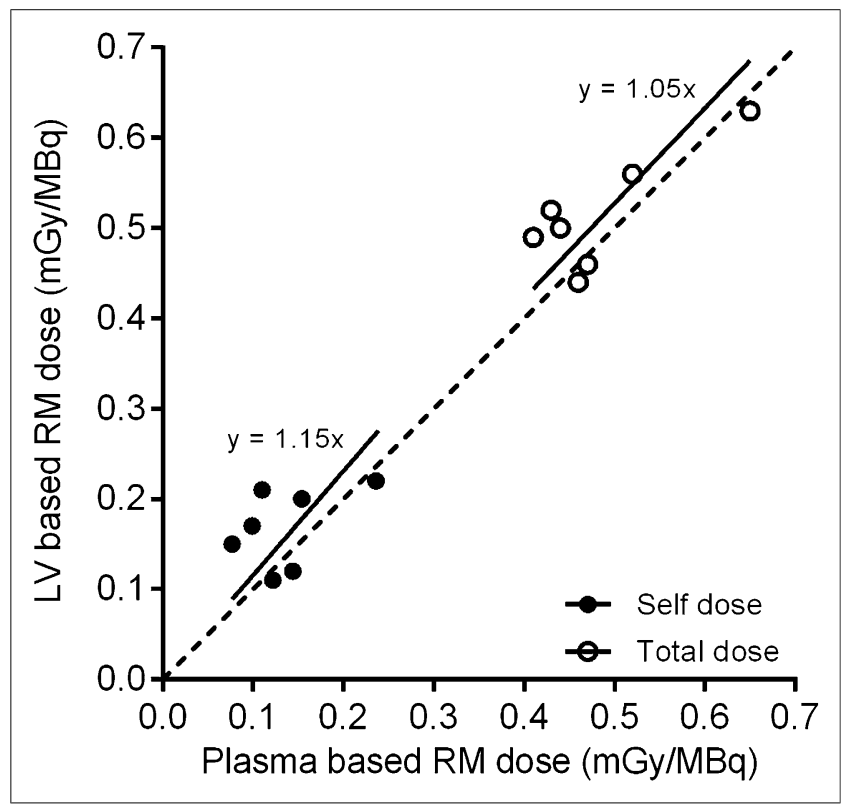

FIGURE 5. RM dose estimates based on plasma and LV approach for self- and total dose in ${ }^{89} \mathrm{Zr}$ PET/CT studies. For radionuclides with little or no long-range photon emission, such as ${ }^{90} \mathrm{Y}$ or ${ }^{177} \mathrm{Lu}$, only self-dose component of overall RM dose should be considered. Relative change in self RM dose between LV-based and plasma-based approaches was $21 \%$ (whereas in total RM dose this difference was diluted due to crossdose contribution, and therefore, the average relative change in total dose was only $6 \%$ ).
The effective dose was calculated to be 0.61 $\pm 0.09 \mathrm{mSv} \cdot \mathrm{MBq}^{-1}$. All possible 3-timepoint combinations were tested in estimating organ-absorbed doses and effective doses. The $1 \mathrm{~h}-48 \mathrm{~h}-144 \mathrm{~h}$ and the $48 \mathrm{~h}-72$ $\mathrm{h}-144 \mathrm{~h}$ protocols showed the smallest $(<4 \%)$ and the largest $(\sim 20 \%)$ discrepancies, respectively, when compared with the 5-timepoint dosimetry protocol (Table 4). Table 5 shows organ effective half-lives of ${ }^{89} \mathrm{Zr}$ cetuximab for 1- to 72-h and 72- to 144-h time intervals. The whole-body effective half-life was $70 \pm 6 \mathrm{~h}$ for the whole imaging range.

\section{DISCUSSION}

This study assessed PET/CT-based biodistribution and dosimetry of ${ }^{89} \mathrm{Zr}$-cetuximab for all organs with positive PET uptake. In addition, an image-based approach for estimating the RM-absorbed dose in ${ }^{89} \mathrm{Zr}$ PET/CT studies was compared with the conventional plasma-based approach.

While ${ }^{18} \mathrm{~F}-\mathrm{FDG}$ is a metabolic tracer that targets tumors in a nonspecific manner, radiolabeled mAbs target a specific tumor cell surface marker. That said, immuno-PET can give insight on tumor targeting and on the amount of the mAb accumulated in the tumor, offering the opportunity to select those patients who will benefit from mAb-based therapy and allowing treatment planning to be tailored to the needs of each patient. More information on the potential added value of immuno-PET in the clinical setting is presented by Wu (16).

The present study showed a nonconstant RMPR over time for ${ }^{89} \mathrm{Zr}$-cetuximab. Hindorf et al. (6) have shown an increasing RMBLR for up to $6 \mathrm{~d}$ after the administration of ${ }^{131}$ I-labeled anti$\mathrm{CD} 22 \mathrm{mAb}$ in patients. Similar findings were reported by Schwartz et al. (5), who found an increasing RMPR with time after radiolabeled antibody administration for patients injected with ${ }^{124} \mathrm{I}-\mathrm{cG} 250$ and ${ }^{124} \mathrm{I}$-huA33. Perk et al. (1) demonstrated approximately 2.5 times higher accumulation of $\mathrm{N}$-sucDf- ${ }^{89} \mathrm{Zr}$ conjugates in bone over time $\left(5.85 \pm 1.05\right.$ percentage injected dose $\left[\%\right.$ ID] $\left.\cdot \mathrm{g}^{-1}\right)$ than of the radioimmunotherapy conjugates in tumor-bearing nude mice studies at $72 \mathrm{~h}$ after injection. This higher accumulation is in agreement with a study by Chang et al. (17), who demonstrated an elevated bone uptake of $5.70 \pm 3.00 \% \mathrm{ID} \cdot \mathrm{g}^{-1}$ at $120 \mathrm{~h}$ after injection. In contrast, the present findings showed a constant RM uptake over time, which could be due to catabolism of cetuximab in the liver. Then the associated ${ }^{89} \mathrm{Zr}$-containing metabolites reenter the bloodstream and they redistribute in the bone marrow. Therefore, the increasing RMPR could be explained, at least in part, by the relative rapid washout of ${ }^{89} \mathrm{Zr}$-cetuximab from the bloodstream in combination with the constant RM uptake. No foci of high activity were detected in bone sites.

The contribution of extrapolations in the cumulated activity before the first and after the last scan was below $20 \%$ as recommended by

TABLE 3

RM-Absorbed Dose

\begin{tabular}{lccc}
\hline Approach & $\begin{array}{c}\text { Self-dose } \\
\left(\mathrm{mGy} \cdot \mathrm{MBq}^{-1}\right)\end{array}$ & $\begin{array}{c}\text { Cross dose } \\
\left(\mathrm{mGy} \cdot \mathrm{MBq}^{-1}\right)\end{array}$ & $\begin{array}{c}\text { Total dose } \\
\left(\mathrm{mGy} \cdot \mathrm{MBq}^{-1}\right)\end{array}$ \\
\hline Plasma & $0.13 \pm 0.05$ & $0.35 \pm 0.03$ & $0.48 \pm 0.08$ \\
LV & $0.17 \pm 0.04$ & $0.34 \pm 0.03$ & $0.51 \pm 0.06$ \\
\hline
\end{tabular}




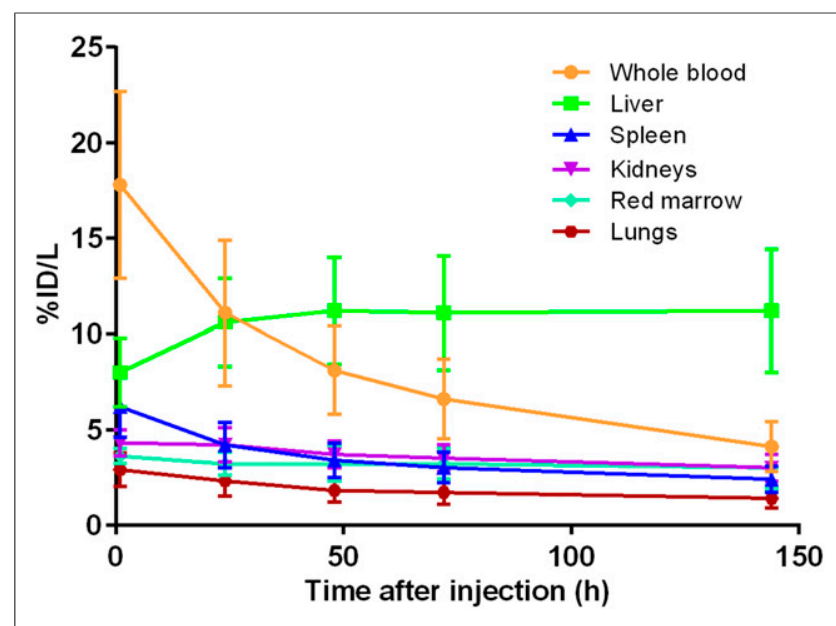

FIGURE 6. Average \%ID per liter as function of time after injection (with decay correction) for all visible organs. Error bars correspond to SD as calculated for 7 patients.

the dosimetry guidelines of the European Association of Nuclear Medicine (18). In addition, the small interpatient variation of the extrapolations (data not shown) implies that the uncertainty due to extrapolations is comparable between patients. Although the wholebody-to-blood cumulated activity ratio decreased, the self RM dose percentage contribution to the total RM dose increased, thus making any variations in parameters related to self RM dose, such as hematocrit and RMECFF, more important.

The estimation of self RM dose as determined with the LV-based approach yielded, on average, $21 \%$ higher values than those obtained with the plasma-based approach. These higher values are due to the constant RMPR (0.19) used in the plasma-based approach. The present findings suggest an increasing RMPR, thus making the latter approach inappropriate. In other words, the relative faster washout of ${ }^{89} \mathrm{Zr}$-cetuximab from the plasma component, compared with the constant uptake in the RM, suggests that the plasma-based approach may not provide for an accurate estimation of RM-absorbed doses. The total RM doses based on plasma and LV approaches were within $6 \%$ of each other. However, for therapeutic analogs with no or little emissions of long-range photons (depending on their energy and half-life) only the self RM dose term is relevant.

The absorbed-dose estimates in the present study are in line (within $20 \%$ for all organs except the liver) with previous ${ }^{89} \mathrm{Zr}$ labeled studies. Rizvi et al. (2) reported that, for ${ }^{89} \mathrm{Zr}$-ibritumomab tiuxetan, the liver was the organ with the highest absorbed dose $\left(1.36 \pm 0.58 \mathrm{mGy} \cdot \mathrm{MBq}^{-1}\right)$, followed by the spleen $(1.04 \pm$ $\left.0.16 \mathrm{mGy} \cdot \mathrm{MBq}^{-1}\right)$, kidneys $\left(0.75 \pm 0.06 \mathrm{mGy} \cdot \mathrm{MBq}^{-1}\right)$, lungs $\left(0.63 \pm 0.11 \mathrm{mGy} \cdot \mathrm{MBq}^{-1}\right)$, and $\mathrm{RM}\left(0.46 \pm 0.05 \mathrm{mGy} \cdot \mathrm{MBq}^{-1}\right)$, whereas the effective dose was found to be $0.55 \pm 0.07 \mathrm{mSv} \cdot \mathrm{MBq}^{-1}$. Borjesson et al. (19) in a radiation dosimetry study of ${ }^{89} \mathrm{Zr}-\mathrm{cmAb}$ U36 found the highest absorbed dose for the liver (1.30 \pm $\left.0.34 \mathrm{mSv} \cdot \mathrm{MBq}^{-1}\right)$, followed by the kidneys $\left(1.00 \pm 0.30 \mathrm{mSv} \cdot \mathrm{MBq}^{-1}\right)$, lungs $\left(0.79 \pm 0.26 \mathrm{mSv} \cdot \mathrm{MBq}^{-1}\right)$, and spleen $(0.72 \pm 0.18$ $\left.\mathrm{mSv} \cdot \mathrm{MBq}^{-1}\right)$. The effective dose was estimated to be $0.60 \pm$ $0.04 \mathrm{mSv} \cdot \mathrm{MBq}^{-1}$. However, a direct comparison of organ-absorbed dose estimates between ${ }^{89} \mathrm{Zr}$-labeled cetuximab and other ${ }^{89} \mathrm{Zr}$ labeled mAbs should be interpreted with care, because metabolism in the liver and specific targeting of each mAb may vary. ${ }^{89} \mathrm{Zr}$ cetuximab is used only for diagnostic purposes, and therefore the effective dose was presented. But in the setting of radioimmunotherapy, the dose on a tumor or the RM should be presented as absorbed dose as well. Because no tumor data are discussed in this article, only RM-absorbed dose data have been reported.

With regards to effective half-lives, only 1 immuno-PET study reports on ${ }^{89} \mathrm{Zr}$ effective half-lives and more specifically in wholebody biologic clearance (20). This was found to be $219 \mathrm{~h}$ on average, and it can be translated to $58 \mathrm{~h}$ on the whole-body effective half-life. This figure is somewhat comparable to the $70 \mathrm{~h}$ seen in the current study. We split the image data points into 2 time intervals to gain insight of organ kinetics over time. With regards to the simplified 3-time-point dosimetry protocol, the first time point $(1 \mathrm{~h})$ is

TABLE 4

Organ-Absorbed Doses

\begin{tabular}{|c|c|c|c|c|c|c|c|c|}
\hline $\mathrm{mGy} \cdot \mathrm{MBq}^{-1}$ & Kidneys & Liver & $\begin{array}{l}\text { Liver excluding } \\
\text { tumor }\end{array}$ & Lungs & Spleen & $\mathrm{RM}^{*}$ & $\begin{array}{l}\text { Whole } \\
\text { body }\end{array}$ & $\begin{array}{l}\text { Effective dose } \\
\left(\mathrm{mSv} \cdot \mathrm{MBq}^{-1}\right)\end{array}$ \\
\hline$M-1$ & 0.82 & 1.54 & 1.61 & 0.50 & 0.79 & 0.46 & 0.45 & 0.52 \\
\hline M-2 & 0.93 & 2.00 & 2.07 & 0.55 & 0.74 & 0.52 & 0.45 & 0.55 \\
\hline$M-3$ & 0.82 & 2.18 & 2.68 & 0.52 & 0.71 & 0.50 & 0.45 & 0.54 \\
\hline$M-4$ & 0.83 & 2.42 & 2.42 & 0.51 & 0.62 & 0.49 & 0.45 & 0.55 \\
\hline $\mathrm{F}-1$ & 1.24 & 2.91 & 2.91 & 0.86 & 1.11 & 0.56 & 0.56 & 0.70 \\
\hline $\mathrm{F}-2$ & 1.32 & 3.48 & 3.67 & 0.80 & 1.10 & 0.44 & 0.56 & 0.71 \\
\hline F-3 & 1.30 & 3.64 & 3.69 & 0.85 & 1.15 & 0.63 & 0.56 & 0.72 \\
\hline \multicolumn{9}{|c|}{ 5-time-point dosimetry approach } \\
\hline Mean & 1.04 & 2.60 & 2.72 & 0.66 & 0.89 & 0.51 & 0.50 & 0.61 \\
\hline SD & 0.24 & 0.78 & 0.78 & 0.17 & 0.22 & 0.06 & 0.06 & 0.09 \\
\hline \multicolumn{9}{|c|}{$\begin{array}{l}\text { Simplified 3-time-point dosimetry } \\
\text { approach }\end{array}$} \\
\hline Mean & 1.04 & 2.50 & - & 0.66 & 0.91 & 0.50 & 0.50 & 0.61 \\
\hline SD & 0.22 & 0.75 & - & 0.17 & 0.22 & 0.06 & 0.06 & 0.09 \\
\hline
\end{tabular}

*Based on manual VOI delineation method. 
TABLE 5

Effective Half-Life (h)

\begin{tabular}{lcc}
\hline Organ & $\begin{array}{c}1-72 \mathrm{~h} \text { after } \\
\text { injection }\end{array}$ & $\begin{array}{c}72-144 \mathrm{~h} \\
\text { after injection }\end{array}$ \\
\hline Kidneys & $60 \pm 10$ & $63 \pm 7$ \\
Liver & $192 \pm 61$ & $79 \pm 9$ \\
Lungs & $41 \pm 7$ & $61 \pm 9$ \\
Spleen & $37 \pm 8$ & $57 \pm 6$ \\
RM & $71 \pm 29$ & $69 \pm 14$ \\
Blood & $30 \pm 3$ & $45 \pm 3$ \\
\hline
\end{tabular}

of importance, because the use of it will lead to more accurate absorbed-dose estimations than when the $24 \mathrm{~h}$ scan is used. In addition, ${ }^{89} \mathrm{Zr}$-labeled mAbs exhibit slow kinetics; thus, targeting of specific organs or tumors will occur in late time points, making the 144-h time point essential in a simplified protocol. The present study suggests that a simplified 3-time-point dosimetry approach may be used for organ-absorbed dose estimation as an alternative to the reference approach, because it yielded similar results (within $\sim 4 \%$ ). This simplified approach will reduce the total scanning time, avoiding unnecessary discomfort and additional radiation burden (due to additional low-dose CT scans) to the patient and without compromising accuracy in dose estimation.

There are technical factors that may hamper accurate quantification of RM activity concentration and thus absorbed-dose estimation. From a technical point of view, partial-volume effect might have resulted in underestimation of RM activity concentrations. On the basis of ${ }^{89} \mathrm{Zr}$ phantom studies (21), the activity concentration of a 2.5$\mathrm{cm}$ sphere surrounded by a homogeneous background can be underestimated by as much as $20 \%$. Nevertheless, the present observation of a nonconstant (increasing) BM-to-background ratio as function of time indicates that partial-volume corrections based on a fixed factor taken from phantom studies (with a sphere-to-background ratio of 10) would provide misleading results. Schwartz et al. (5) used recovery coefficients for partial-volume correction derived from phantom studies. Unfortunately, there was no report on how the BM-to-background ratio behaved over time, because a nonconstant ratio would require a time-varying partial-volume correction. Notably, the current study showed small deviations in $\mathrm{AC}_{\mathrm{RM}}$ while varying the VOIs, indicating a minimal impact of the partial-volume effect. In addition, the 6-mL VOIs were used on the LV segments such that a distance of at least $1 \mathrm{~cm}(\sim 2 \times$ scanner spatial resolution) from the outer $\mathrm{LV}$ bone was ensured. In any case, even if partial-volume corrections were applied, it would only increase the dissociation of RM dose estimation between image- and plasma-based approaches.

\section{CONCLUSION}

Total RM dose estimates derived from plasma- and imagebased approaches are equal within $6 \%$. For dosimetry purposes in immuno-PET this would be acceptable. Nevertheless, an imagebased approach, using manual delineation of the $\mathrm{LV}$, is preferred for determining RM dose estimates, because it accounts for a nonconstant RMPR. The liver showed the highest absorbed dose among all organs, and the effective dose was $0.61 \pm 0.09 \mathrm{mSv} \cdot \mathrm{MBq}^{-1}$. A simplified approach using 3 time points appears to be feasible, reducing logistical costs and scanning time required.

\section{DISCLOSURE}

The costs of publication of this article were defrayed in part by the payment of page charges. Therefore, and solely to indicate this fact, this article is hereby marked "advertisement" in accordance with 18 USC section 1734 . The study was financially supported in part by Philips Healthcare. No other potential conflict of interest relevant to this article was reported.

\section{REFERENCES}

1. Perk LR, Visser GW, Vosjan MJ, et al. ${ }^{89} \mathrm{Zr}$ as a PET surrogate radioisotope for scouting biodistribution of the therapeutic radiometals ${ }^{90} \mathrm{Y}$ and ${ }^{177} \mathrm{Lu}$ in tumorbearing nude mice after coupling to the internalizing antibody cetuximab. $\mathrm{J} \mathrm{Nucl}$ Med. 2005;46:1898-1906.

2. Rizvi SN, Visser OJ, Vosjan MJ, et al. Biodistribution, radiation dosimetry and scouting of ${ }^{90} \mathrm{Y}$-ibritumomab tiuxetan therapy in patients with relapsed B-cell non-Hodgkin's lymphoma using ${ }^{89} \mathrm{Zr}$-ibritumomab tiuxetan and PET. Eur J Nucl Med Mol Imaging. 2012;39:512-520.

3. Koi L, Bergmann R, Bruchner K, et al. Radiolabeled anti-EGFR-antibody improves local tumor control after external beam radiotherapy and offers theragnostic potential. Radiother Oncol. 2014;110:362-369.

4. Sgouros G. Bone marrow dosimetry for radioimmunotherapy: theoretical considerations. J Nucl Med. 1993;34:689-694.

5. Schwartz J, Humm JL, Divgi CR, Larson SM, O’Donoghue JA. Bone marrow dosimetry using ${ }^{124}$ I-PET. J Nucl Med. 2012;53:615-621.

6. Hindorf C, Linden O, Tennvall J, Wingardh K, Strand SE. Time dependence of the activity concentration ratio of red marrow to blood and implications for red marrow dosimetry. Cancer. 2002;94(4, suppl);1235-1239.

7. Zhou Y, Baidoo KE, Brechbiel MW. Mapping biological behaviors by application of longer-lived positron emitting radionuclides. Adv Drug Deliv Rev. 2013;65:1098-1111.

8. Nagengast WB, de Vries EG, Hospers GA, et al. In vivo VEGF imaging with radiolabeled bevacizumab in a human ovarian tumor xenograft. J Nucl Med. 2007;48:1313-1319.

9. Oude Munnink TH, Arjaans ME, Timmer-Bosscha H, et al. PET with the ${ }^{89} \mathrm{Zr}$ labeled transforming growth factor-beta antibody fresolimumab in tumor models. J Nucl Med. 2011;52:2001-2008.

10. Surti S, Kuhn A, Werner ME, Perkins AE, Kolthammer J, Karp JS. Performance of Philips Gemini TF PET/CT scanner with special consideration for its time-offlight imaging capabilities. J Nucl Med. 2007;48:471-480.

11. Stabin MG. MIRDOSE: personal computer software for internal dose assessment in nuclear medicine. J Nucl Med. 1996;37:538-546.

12. Greuter HN, Boellaard R, van LA, Franssen EJ, Lammertsma AA. Measurement of ${ }^{18} \mathrm{~F}-\mathrm{FDG}$ concentrations in blood samples: comparison of direct calibration and standard solution methods. J Nucl Med Technol. 2003;31:206-209.

13. Shen S, Denardo GL, Sgouros G, O'Donnell RT, DeNardo SJ. Practical determination of patient-specific marrow dose using radioactivity concentration in blood and body. J Nucl Med. 1999;40:2102-2106.

14. Stabin MG, Siegel JA, Sparks RB. Sensitivity of model-based calculations of red marrow dosimetry to changes in patient-specific parameters. Cancer Biother Radiopharm. 2002;17:535-543.

15. Beddoe AH, Darley PJ, Spiers FW. Measurements of trabecular bone structure in man. Phys Med Biol. 1976;21:589-607.

16. Wu AM. Antibodies and antimatter: the resurgence of immuno-PET. J Nucl Med. 2009;50:2-5.

17. Chang AJ, De Silva RA, Lapi SE. Development and characterization of ${ }^{89} \mathrm{Zr}$ labeled panitumumab for immuno-positron emission tomographic imaging of the epidermal growth factor receptor. Mol Imaging. 2013;12:17-27.

18. Hindorf C, Glatting G, Chiesa C, Linden O, Flux G. EANM Dosimetry Committee guidelines for bone marrow and whole-body dosimetry. Eur J Nucl Med Mol Imaging. 2010;37:1238-1250.

19. Börjesson PK, Jauw YW, de Bree R, et al. Radiation dosimetry of ${ }^{89} \mathrm{Zr}$-labeled chimeric monoclonal antibody U36 as used for immuno-PET in head and neck cancer patients. J Nucl Med. 2009;50:1828-1836.

20. Pandit-Taskar N, O'Donoghue JA, Beylergil V, et al. ${ }^{89} \mathrm{Zr}$-huJ591 immuno-PET imaging in patients with advanced metastatic prostate cancer. Eur J Nucl Med Mol Imaging. 2014;41:2093-2105.

21. Makris NE, Boellaard R, Visser EP, et al. Multicenter harmonization of ${ }^{89} \mathrm{Zr}$ PET/CT performance. J Nucl Med. 2014;55:264-267. 